Gdańsk 2019, Nr. 40

https://doi.org/10.26881/sgg.2019.40.04

Christian Zehnder

Université de Fribourg / Uniwersytet we Fryburgu

https://orcid.org/0000-0003-4442-0093

\title{
Sprachkrise, Epiphanie, Flimmern: Von Hofmannsthal zu Vvedenskij. Ein Vorschlag
}

Das Schreiben des russischen Dichters Aleksandr Vvedenskij (1904-1941) ist eminent sprachkritisch und kulminiert im „ästhetischem Schein“ erratischer Symbole. Dennoch lässt es sich nicht als Wechselspiel von Krise und Epiphanie beschreiben, wie eine Gegenüberstellung von Vvedenskijs Grauem Heft (1932/33) mit Hugo von Hofmannsthals Ein Brief (1902) zeigt. Die „Unverbundenheit der Welt“, so die These des Artikels, ist bei Vvedenskij der Sprachkrise vorgeordnet. Von der stummen Phänomenalität der Dinge her ist daher anders als bei Hofmannsthal keine noch so momenthafte neue Sinnstiftung möglich. Auf der anderen Seite kann nach Vvedenskij selbst eine radikal-avantgardistische Demonstration der Sprachinsuffizienz dem „Zerfall“ der Welt nicht gerecht werden. Statt nur von einer sprachspielerischen oder epiphanischen könnte man insofern von einer katastrophalen Moderne sprechen.

Schlüsselwörter: Moderne, Sprachkrise, Epiphanie, Plötzlichkeit, Absurdismus, Hugo von Hofmannsthal, Aleksandr Vvedenskij

Language Crisis, Epiphany, Flicker: From Hofmannsthal to Vvedensky. A Suggestion. The writing of the Russian poet Aleksandr Vvedensky (1904-1941) is deeply critical of language and it culminates in the "aesthetic appearance" of erratic symbols. Nevertheless, it cannot be described as a typical high modernist interplay of crisis and epiphany, as a juxtaposition of Vvedensky's Grey Notebook (1932/33) with Hugo von Hofmannsthal's A Letter (1902) shows. The "disconnectedness of the world," this essay argues, is for Vvedensky prior to the language crisis. In contrast to Hofmannsthal, the mute phenomenality of things, therefore, does not allow for even a momentary new integration. On the other hand, even a radical Avant-garde enactment of the insufficiency of language cannot do justice to that disintegration of the world. With regard to Vvedensky, one coud speak, then, of a catastrophic than linguistic or epiphanic Modernism.

Keywords: Modernism, language crisis, epiphany, suddenness, absurdism, Hugo von Hofmannsthal, Alexandr Vvedensky

Das Paradigma der modernen ,Sprachkrise beschreibt ein Auseinanderfallen von Welt und Sprache: Die Destabilisierung kohärenter Weltbilder (philosophischer, wissenschaftlicher, religiöser) stellte die Möglichkeit einer sprachlichen Erfassung der Wirklichkeit überhaupt in Frage. Die Durchtrennung des vertrauenswürdigen Bands zwischen Welt und Sprache 
wurde so zum Signum der literarischen Moderne schlechthin. Das heißt aber auch: Das Problem der Wirklichkeitsdarstellung blieb aktuell, es verschob sich nur auf eine andere Ebene. Dem alten Mimesisproblem erwuchs ein neuer, negativer Bezug. Es ging der Literatur seit dem späten 19. Jahrhundert nicht mehr darum, eine adäquate Sprache für die Wirklichkeit zu finden, sondern für die Krise ihrer Sagbarkeit. Wenn die Literatur in der Moderne in manchen Fällen eminent selbstbezüglich ist, so bedeutet dies grundsätzlich keine Abkapselung von der ,Außenwelt', sondern gerade umgekehrt eine verschärfte Konfrontation mit ihr, oder eben: mit dem Verlust dieser Außenwelt als einem geordneten, vor-bildlichen Gefüge. Bezeichnenderweise schrieben sich die europäischen Avantgarden, so sehr sie sich - falls es denn noch nötig war - der Austreibung des 19. Jahrhunderts annahmen, nicht Anti-Realismus, Phantastik und dergleichen mehr auf die Fahnen, sondern einen höheren, realeren, „supremen“ "Realismus. ${ }^{1}$

Als Urszene und gültiger Ausdruck der Sprachkrise galt im deutschsprachigen Raum lange Zeit und in mancher Hinsicht bis heute Hugo von Hoffmannsthals Ein Brief (1902), jenes auf den 22. August 1603 datierte Bekenntnisschreiben des fiktiven Lord Chandos an Francis Bacon über das Verstummen. "Es ist mir völlig die Fähigkeit abhanden gekommen“, schreibt Lord Chandos, ,über irgend etwas zusammenhängend zu denken oder zu sprechen“ (Hoffmannsthal 1991a: 48). Und es folgt die immer wieder zitierte Stelle: „[...] die abstrakten Worte, deren sich doch die Zunge naturgemäß bedienen muß, um irgendwelches Urteil an den Tag zu geben, zerfielen mir im Munde wie modrige Pilze" (Hoffmannsthal 1991a: 48-49). Wie mit der Zeit ins Bewusstsein rückte, beschreibt dieser Satz zwar eine Krise der Sprache, ist von ihr aber selbst offensichtlich nicht betroffen. Die Bildung von abstracta funktioniert klaglos und namentlich das, was man als ,metaphorische Rationalität' bezeichnen könnte, behauptet sich unversehrt: Kann man sich eine treffendere, eine kohärentere Metapher als jene der „modrigen Pilze“ vorstellen? Nicht zu Unrecht bezeichnete Joachim Kühn (1975, 24, 29; zit. nach Merger 1995, 15, Fußnote 47) Hofmannsthals Text als „sprachlich virtuose Darstellung von Sprachzerfall“" und las ihn gegen den Strich gar als Parodie der Sprachskepsis Fritz Mauthners mit ihrem Pathos des Verstummens. Günter Saße (1977; zit. nach Merger 1995, 16-18) hat die Sprachkrise in ihrer Fin-de-siècle-Ausprägung als „sprachtraditionell“ bezeichnet: Hier wird eine Sprache für die Krise gefunden, mehr noch: das Benennen der Dysfunktionalität wird geradezu zum Medium eines Triumphs der Sprache. Die Krisendiagnostik ist insofern eine Strategie der Entautomatisierung, eine Art Verpuppung, aus der die poetische Sprache am Ende wie ein frisch geschlüpfter Schmetterling hervorgeht. Von der „sprachtraditionellen“ unterscheidet Saße eine „sprachdemonstrative“ Auffassung der Krise, wie er sie erst für spät- und neo-avantgardistische Poetiken in Anschlag bringt. Der Umstand, dass Sprachzerfall qua intakte Sprache nicht darstellbar ist, ja streng genommen unbeweisbar bleibt, wird hier performativ umgesetzt, d. h. die fehlgehende Äußerung wird auch als Defekt präsentiert. Für die derart zur permanenten Krise entfaltete Sprachskepsis - als Defekt

Zum Verhältnis von Abstraktion und Realismus in der Avantgarde s. ausführlich Zimmermann 2007.

2 Für die Zwecke der vorliegenden Gegenüberstellung beschränke ich mich auf einige ausgewählte Positionen aus der kaum überschaubaren Fülle an Literatur zu Hofmannsthals Brief. Für eine umfassende Relektüre s. Günther 2004, für eine neue komparatistische Perspektive Majewski 2018. 
Selbstdesillusionierung von Sinnstiftung - steht idealtypisch das Werk von Samuel Beckett (s. Merger 1995).

Das so verstandene Paradigma der Sprachkrise bezieht sich jeweils auf einen Sprecher, der in seinen Mitteilungsversuchen scheitert. Es wird also eine Norm gelingender Kommunikation supponiert, aus welcher der moderne Künstler in seiner Kondition der ,metaphysischen Obdachlosigkeit' herausfällt, herausfallen muss. Der Rede von der Sprachkrise liegen insofern spezifisch logisch-positivistische Prämissen zugrunde, denn im Zentrum steht die Unterscheidung, was unter welcher Voraussetzung als, sagbar' oder, unsagbar' gelten kann. Eine solche Unterscheidung ist zunächst durchaus im Sinne von Hofmannsthals Brief: Lord Chandos stellt sich auf die Seite der Unsagbarkeit, und dies gegenüber Francis Bacon, dem Begründer der neuzeitlich-empirischen Naturwissenschaften, der die Haltung verkörpert, dass die Welt - einschließlich mythischer Überlieferungen - auf Grund ihrer Daten grundsätzlich les- und aufschließbar sei. ${ }^{3}$ Der springende Punkt ist jedoch, dass Hofmannsthals Text bei dieser Unterscheidung nicht stehen bleibt, und auch nicht beim Selbstwiderspruch des eloquenten Sprechens über den Verlust der Fähigkeit zu sprechen. Vielmehr führt die Sprachkrise des Lord Chandos zu einer neuen Sicht der Welt, die man am treffendsten als epiphanisch beschreiben kann. Die Krise von Hofmannsthals Briefschreiber auf eine sprachliche zu reduzieren heißt, die ihn nun überwältigende phänomenale Evidenz von Dingen und Alltagshandlungen auszublenden. Darauf hat Karl Heinz Bohrer hingewiesen. ${ }^{4}$ Stabil-kontinuierliche Weltkonstruktionen werden von einem Augenblickserleben abgelöst, durch welches zwar keine Sinnganzheit wiedergewonnen wird, aber sehr wohl die Möglichkeit von Partizipation an einer gesteigerten Intensität: an einer ,,̈̈berschwellenden Flut höheren Lebens“ (Hofmannsthal 1991a: 50). Keiner der ihn umgebenden Menschen, schreibt Lord Chandos, bemerke,

daß mein Auge lange an den häßlichen jungen Hunden hängt oder an der Katze, die geschmeidig zwischen Blumenscherben durchkriecht, und daß es unter allen den ärmlichen und plumpen Gegenständen einer bäurischen Lebensweise nach jenem einen sucht, dessen unscheinbare Form, dessen von niemand beachtetes Daliegen oder -lehnen, dessen stumme Wesenheit zur Quelle jenes rätselhaften, wortlosen, schrankenlosen Entzückens werden kann. (Hofmannsthal 1991a: 53)

Lord Chandos sucht die Epiphanie - das Aufscheinen des Unscheinbaren -, und doch bleibt sie notwendig unvorhersehbar, sie ereignet sich „,in irgend einem Moment, den herbeizuführen auf keine Weise in meiner Gewalt steht" (Hofmannsthal 1991a: 50). Er schreibt vom Schweigen, um für die eminent ausdruckslosen Dinge Raum zu schaffen und ihrer Sprachlosigkeit gerecht zu werden. Dass dieses Schweigen aber noch einmal ein Modus der Sprache ist, einer neuen Bildlichkeit, versteht sich gewissermaßen von selbst. Logische Schranken, wie sie etwa die von Wittgenstein geprägte Neoavantgarde der 1960er Jahre umtreiben werden, spielen hier keine signifikante Rolle.

3 Die Referenzen an Bacon im Brief untersuchte Schultz 1961. Zur „Interpretation bestimmter antiker Mythen als Metaphern abstrakter Begriffe" in Bacons The Wisdom of the Ancients (1609) vgl. die Erläuterungen in Hofmannsthal 1991: 279-280.

4 Bohrer 1998 [1981]: 55-63, bes. 58. Bohrer bespricht hier narrative Realisierungen des epiphanischen Augenblicks in Erzählungen des frühen Hofmannsthal. 
Ryszard Nycz spricht treffend und mit ausdrücklichem Bezug auf Hofmannsthal vom Übergang „einer Ästhetik des Unaussprechlichen zu einem epiphanischen Werkbegriff“" in der europäischen Moderne. Paul de Man (1960: 75-76) wies bereits für die vermeintlich objektlose, ausschließlich auf die eigene Struktur bezogene poésie pure Stéphane Mallarmés - eines zentralen Autors für die Genese der Sprachkrise - einen Drang zum „natürlichen Objekt“ und letztlich eine „Suprematie des natürlichen Seins“ nach. Um seine These zu belegen, verweist de Man (1960:76) auf die Naturbilder, wie sie in Mallarmés Lyrik quasi seriell auftreten - Meer, Vogel, Flügel, die Jahreszeiten, Nacht, Sonne -, und er führt aus:

So körperlos diese Bilder wirken und obwohl sie idealisiert und universalisiert erscheinen, sind sie doch nie nur ideales und universelles Bild des Wirklichen und des Singulären. Das Meer, der Vogel, das Sternbild treiben und ziehen die Lyrik Mallarmés an wie ein Meer, ein Vogel und ein Sternbild, von der Erde aus betrachtet: Das Meer verschlingt uns, der Vogel verführt uns durch die Leichtigkeit seines Flugs, das Sternbild durch seine unveränderliche Ordnung.

Nun ist offensichtlich, dass Mallarmés Naturbilder, so wie de Man sie liest, keine epiphanischen in Hofmannsthals Sinne sind. Dafür sind sie zu wenig unmittelbar, zu distanziert, und es geht ihnen die Unver häl t n is mäßigke it ab, die charakteristisch ist für die Faszination der Blumenscherben und so vieler anderer alltäglich unbemerkter Dinge im Brief des Lord Chandos. Die moderne Epiphanie ist immer auch eine Vergrößerung, eine sich aufdrängende Überhöhung von Lapidarem zu einer Vision, zu einer profanen ,Erleuchtung (,alles dies kann das Gefäß meiner Offenbarung werden“; Hofmannsthal 1991a: 50). Mallarmés Naturbilder sind dagegen grundsätzlich noch klassisch-romantisch aufgeladen, wenn auch ihre Entrücktheit sie als neutralisiert, erkaltet erscheinen lässt.

De Mans „terrestrische" Lesart Mallarmés legt nahe, dass es einen Umgang mit der Sprachkrise gibt, der weder ,sprachphilosophisch' noch epiphanisch wäre. Ich meine, dass ein solches Weder-Noch kennzeichnend ist für das Werk des spätavantgardistischen russischen Dichters Aleksandr Vvedenskij (1904-1941). Vvedenskij kann mit seinem schmalen Werk zweifellos als einer der radikalsten Vertreter der sprachkritischen Moderne gelten, und zugleich entzieht er sich auf merkwürdige Weise dem etablierten literaturwissenschaftlichen Paradigma der Sprachkrise. ${ }^{6}$ Abgesehen von seinen teilweise noch futuristischen Anfängen ist Vvedenskij nicht sprach-zerstörerisch und nicht ikonoklastisch, ja er greift zurück auf ein Repertoire relativ weniger stehender, auratisch wirkender Symbole - u. a. Meer, Fluss, Vogel, wildes Tier -, das mit jenem Mallarmés teilweise identisch ist. Was Vvedenskijs „Hieroglyphen“7 mit Mallarmés Naturbildern tatsächlich (typologisch) verbindet, ist der Umstand, dass sie scheinbar keine subjektive Färbung haben und eine ausgesprochen starke Anziehungskraft ausüben, zu der es aber keinen erlebnismäßigen Zugang gibt. ${ }^{8}$ Vvedenskijs Hieroglyphe ist

Nycz 2001: 41-47. Alle Übersetzungen stammen von mir - Ch. Z.

6 Für einen knappen Überblick zu Vvedenskij auf Deutsch s. Zehnder 2013.

Vgl. zu dem von Leonid Lipavskij eingeführten Begriff der absurdistischen Hieroglyphe Gerasimova 2011: 19-24

8 Eine Art ungeerdeter poésie pure unterstellte dem frühen Vvedenskij der weniger radikale Nikolaj Zabolockij (2011 [1926]: 395): „Ihre Verse stehen nicht auf der Erde. [...] Funkelnde Steine fliegen hier umher und seltsame Klänge sind zu hören - aus der Leere; eine Widerspiegelung nichtexistierender Welten.“ 
wesentlich und von vornherein unentzifferbar (anders als jene Hofmannsthals, auf die ich noch zu sprechen kommen werde). Sie interagiert sinn-los mit anderen, ihr benachbarten Hieroglyphen.

Im Folgenden werde ich Vvedenskijs sprachkritische Stellung anhand seines Grauen Hefts (Seraja tetrad', 1932/33), eines Fragments, bestehend aus Gedicht- und Prosateilen, herausarbeiten und in paralleler Lektüre mit Hofmannsthals Brief diskutieren. Wenn ich den russischen Symbolismus als Bezugsgröße so quasi überspringe, soll damit keineswegs gesagt sein, dass eine Parallellektüre mit solchen Autoren wie Innokentij Annenskij, Valerij Brjusov oder noch Aleksandr Blok nicht angezeigt wäre. ${ }^{9}$ Im Gegenteil; in jüngerer Zeit ist die Grenze zwischen dem Symbolismus und der Avantgarde zunehmend als fließend beschrieben worden. Oleg Kling (1999) hat von einer „latenten Existenz“ des Symbolismus nach seinem Ende gesprochen und rief so im Grunde eine literaturgeschichtliche Selbstverständlichkeit in Erinnerung, die eher aus Gründen institutioneller Akzentsetzungen teilweise aus dem Blick geraten war. ${ }^{10}$ Mir geht es bei der Parallellektüre von Vvedenskijs Grauem Heft mit Hofmannsthals Brief freilich nicht um Nuancen der Periodisierung zwischen Symbolismus und Avantgarde. Vielmehr möchte ich anhand dieses eng eingegrenzten Korpus die Frage nach dem Verhältnis der sprachkritischen zur epiphanischen und schließlich zu einer nicht-mehr-epiphanischen Moderne thematisieren. Mein Vorschlag ist also, das Phänomen der radikalen Sprachkrise bei Vvedenskij durch die Folie von Hofmannsthals ,klassisch-modernem' Brief aus einem vornehmlich russischen Kontext herauszulösen und stattdessen in eine transnationale Konstellation zu bringen.

Zur Verbreitung von Vvedenskijs Werk im Westen ist vorauszuschicken: Zwar liegen Übersetzungen Vvedenskijs in mehrere Sprachen vor ${ }^{11}$, doch ist er in den westlichen Literaturwissenschaften, auch in der Slavistik, als eigenständiger Autor bis heute wenig bekannt. Als Teil der absurdistischen Spätavantgarde bzw. in Bezug auf das „Ende der russischen Avantgarde“ (Jean-Philippe Jaccard) ist Vvedenskij dagegen in jüngerer Zeit vermehrt Gegenstand der Forschung geworden. ${ }^{12}$ In Russland bleibt er ein poet's poet - vielleicht der einflussreichste überhaupt neben Velimir Chlebnikov (welchem Majakovskij (1959 [1922]: 23) diesen Ehrentitel zugewiesen hatte: poèt dlja proizvoditelja: „Dichter für den Produzenten“). ${ }^{13}$ Anders als Chlebnikovs utopische „Sternensprache“ (zvezdnyj jazyk) ist Vvedenskijs „Stern der Sinnlosigkeit" (zvezda bessmyslicy $\left.{ }^{14}\right)$ allerdings in der russischen Philologie auch eher sporadisch, jedenfalls in einem stark spezialistischen Diskurs untersucht geworden. ${ }^{15}$

\footnotetext{
9 Eine umfassende Darstellung der Motive des Schweigens, Verstummens und der Sprachlosigkeit im frühen, „diabolischen“ russischen Symbolismus gibt Hansen-Löve 1989.

10 S. vor allem auch den Sammelband Kovalenko 2003 über „Symbolismus in der Avantgarde“.

11 U. a. Urban 1992a; Urban 1992b; Abril 2012; Ostashevsky 2006; Vviedenskij 2013 b.

12 Erwähnt werden können etwa Müller 1978; Jaccard 1991; Hansen-Löve 1994; Hansen-Löve 1998; Sauerwald 2010; Schloßberger-Oberhammer 2018.

13 Neuere Beispiele für den ,Vvedenskij der Dichter` sind Martynova 2019 [2008], Barskova 2011 und Skidan 2013

14 Vgl. Jakov Druskins (2000 [1973]) bis heute prägende Vvedenskij-Lektüre Zvezda bessmyslicy.

15 S. etwa den Sammelband Ičin/Kudrjavcev 2006 und die großangelegte Untersuchung Valieva 2007. Eine weit größere Leserschaft in Russland wie im Westen, akademisch wie nicht-akademisch, hat Vvedens-
} 
In den von Leonid Lipavskij aufgezeichneten Gesprächen (Razgovory, 1933/34) der absurdistischen Dichtervereinigung Obèriu (Ob"edinenie real'nogo iskusstva - Vereinigung für Reale Kunst $\left.{ }^{16}\right)$ verlautbart Vvedenskij programmatisch:

Ich verübte einen Übergriff auf Begriffe, auf ihre basalen Verallgemeinerungen, was vor mir noch keiner getan hatte. So führte ich gleichsam eine poetische Kritik der Vernunft durch - eine grundlegendere als jene, abstrakte. Ich zog in Zweifel, dass beispielsweise Haus, Datscha und Turm durch den Begriff „Gebäude“verbunden sind. Womöglich muss Schulter mit Vier verbunden werden. Ich tat dies in der Praxis, in der Poesie, und bewies es so. Ich überzeugte mich von der Verlogenheit der bisherigen Verbindungen, aber ich kann nicht sagen, welche neuen an ihre Stelle treten sollen. Ich weiß nicht einmal, ob es ein System von Verbindungen geben sollte oder mehrere. Und ich habe ein Grundgefühl von der Unverbundenheit der Welt und der Fragmentiertheit der Zeit. Da dies aber der Vernunft widerspricht, heißt das: die Vernunft hat kein Verständnis von der Welt. ${ }^{17}$

Man sieht unschwer, wie nah Vvedenskij hier Hofmannsthal (dessen Werk er aller Wahrscheinlichkeit nach nicht kannte und zu dem sein Schreiben keinerlei direkte Verbindungen aufweist) in der Sache kommt. Im Brief des Lord Chandos heißt es:

Es gelang mir nicht mehr, sie [Menschen und Handlungen] mit dem vereinfachenden Blick der Gewohnheit zu erfassen. Es zerfiel mir alles in Teile, die Teile wieder in Teile, und nichts mehr ließ sich mit einem Begriff umspannen. Die einzelnen Worte schwammen um mich; sie gerannen zu Augen, die mich anstarrten und in die ich wieder hineinstarren muß: Wirbel sind sie, in die hinabzusehen mich schwindelt, die sich unaufhaltsam drehen und durch die hindurch man ins Leere kommt. (Hofmannsthal 1991a: 49)

In beiden Fällen verliert die Sprache ihr routinemäßiges Vermögen, Konzepte über mehrere Phänomene zu prädizieren, d. h. diese klassifizierend zu „verbinden“ (Vvedenskij) bzw. zu „umspannen“(Hofmannsthal). Was ist geschehen? Lord Chandos beschreibt in seiner Selbstdiagnose einen Verlust jeglicher Distanznahme gegenüber der Welt; die Wörter und Dinge hätten sich ihm vereinzelt dadurch, dass er sie je nur noch in einer „unheimlichen Nähe“ wahrnehmen konnte, als würden sie sich in geradezu obszöner Vergrößerung zeigen, wie unter einer Lupe. Interessanterweise ist der Moment der Distanzlosigkeit - hier als Krankheitsbild dargestellt - aber auch schon Teil der Lösung. Denn in Form unmittelbaren Erlebens wird Nähe gegen Ende des Briefs zur zentralen Qualität der Epiphanie (oder „Offenbarung“, wie Chandos schreibt). Ebenso kehren die „Wirbel“, zunächst Krisenzeichen, in den epiphanischen Augenblicken positiviert wieder:

Und das Ganze ist eine Art fieberisches Denken, aber Denken in einem Material, das unmittelbarer, flüssiger, glühender ist als Worte. Es sind gleichfalls Wirbel, aber solche, die nicht wie die Wirbel der Sprache ins Bodenlose zu führen scheinen, sondern irgendwie in mich selber, und in den tiefsten Schoß des Friedens. (Hofmannsthal 1991a: 54)

kijs Mitstreiter Daniil Charms. Charms ist auch bereits Gegenstand komparatistischer Arbeiten geworden; so wurde er ausführlich mit Beckett oder mit Kafka verglichen (s. Tokarev 2002 und Lehmann 2013).

16 Obèriu wurde 1927 von Vvedenskij und Charms ausgerufen. Dem Kreis gehörten nebst den bisherigen Činari - den Schulfreunden Aleksandr Vvedenskij, Jakov Druskin und Leonid Lipavskij - u. a. die Dichter Konstantin Vaginov und Nikolaj Zabolockij an.

17 Lipavskij 2000 [1933/34]: 186. S. Peter Urbans (1992a: 110-118) Übersetzung (von der meine an einigen Stellen deutlich abweicht). 
Die Krise enthält in diesem Sinne bei Hofmannsthal schon die Heilung. Aus seinem distanzierten Weltverständnis, das er mit Francis Bacon teilte, verfällt Chandos zunächst in ein krankhaftes Nähe-Verhältnis zur Welt. Zu „schweigen“ wird für ihn aber in einem zweiten Schritt bedeuten, sich mit der Nähe zu versöhnen. Was heißt das? Hilfreich ist in diesem Zusammenhang das Bild vom Vergrößerungsglas: Was die neue, verwandelte Nähe von der pathologischen unterscheidet, ist der Wegfall instrumenteller, manipulativer, Skalierung'. Auf die Epiphanie muss Lord Chandos zwar durchaus entbehrungsreich warten, am Ende stellt sie sich aber - falls sie sich denn einstellt - selbst ein, sie bringt ihm Fülle.

Wenn Aleksandr Vvedenskijs Irrewerden an Verallgemeinerungen ein ganz anderes ist, so hat dies offenbar damit zu tun, dass sein Schreiben keinen emphatischen Begriff von Unmittelbarkeit, keine transformierte, keine erfüllte Nähe zur Welt kennt. Doch bereits der Ursprung der Krise ist ein anderer: Während Hofmannsthal sie erleidet, behauptet Vvedenskij, die Krise bewusst herbeigeführt zu haben. Interessanterweise setzt die Krise des Lord Chandos ein mit dem Plan, im Geiste von Francis Bacons Wisdom of the Ancients „die Fabeln und mythischen Erzählungen [...] auf[zu]schließen als die Hieroglyphen einer geheimen, unerschöpflichen Weisheit" (Hofmannsthal 1991a: 46-47). Sein Anspruch auf entzifferndes Verstehen lässt den Lord Chandos zuallererst taumeln und ins Leere laufen. Vvedenskij unternimmt einen solchen Versuch des Entzifferns von Hieroglyphen geradezu demonstrativ gar nicht erst. Er stellt seine Hieroglyphen vielmehr mit „transgressiver Geste“ (Podoroga (1993: 149) über Obèriu) in den Raum. Konsequenterweise kennt Vvedenskij auch kein Pathos des Verstummens. Er gibt sich in seiner Geste vielmehr betont selbstbewusst. Begriffsklassen habe er poetisch auseinandergehauen.

Der Satz „Womöglich muss Schulter mit Vier verbunden werden“ ist eine Kurzformel für seine Poetik, in der die vereinzelten Wörter neu reagieren und interferieren. Im Grauen Heft heißt es etwa: „Haus Wald Himmel, als wären sie Mongolen, / wurden auf einmal von

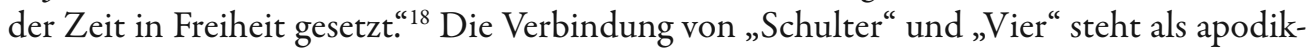
tische Behauptung eines unerschrockenen Pioniers der poetischen „Praxis“ im Raum. Und doch ist der Modus seiner prophetischen Rede das „womöglich“. Die Kunst kann beweisen, dass es neue Verbindungen braucht, und Vvedenskij hat den Beweis, wie er sagt, mit seiner Poesie erbracht. Doch wie weit reicht die Autorität dieser „poetischen Kritik der Vernunft“? Besonders wichtig scheint mir folgende Bemerkung Vvedenskijs in den Gesprächen: „Wie jammerschade, sie [die Kunst] ist subjektiv. Die Poesie stellt bloß Wortwunder her, keine echten. Ja, und wie man die Welt wieder zusammensetzen soll, ist nicht bekannt" (Lipavskij 2000 [1933/34]: 186). In seinen poetischen Taten, im „praktischen“ Nachweis der Ungültigkeit sprachlich sanktionierter Logik sieht Vvedenskij demnach kein echtes Wunder. Die Krise der Welt und die Krise der Sprache sind hier anders als etwa bei Fritz Mauthner (s. Merger 1995: 20) nicht symmetrisch, sie sind nicht komplementär einander zugeordnet. Dem Auseinanderfallen der Welt steht bei Vvedenskij nicht homolog das sprachliche Krisenereignis gegenüber. Vielmehr muss hier die Logik aktiv aus dem Weg geräumt werden, sie muss „die Kontrolle über die Welt verlieren“ (Podoroga 1993: 147), damit ein offener Blick auf die Unverbundenheit quasi nackt: katastrophal möglich wird.

18 Vvedenskij 1993a: 77. S. Urbans (1992a: 26-33) Übersetzung (von der meine wiederum abweicht). 
Eine Rekonfiguration kann also von der poetischen Sprache geleistet werden, aber sie bleibt dabei notwendig ein „Wortwunder“. Das Warten auf ein „echtes Wunder“ dauert an. Das heißt: Letztlich führt bei Vvedenskij kein Weg zu den Epiphanien. Moderne Epiphanien sind, so viel Verlorenes, Verschüttetes, Entzaubertes sie auch bezeugen, noch immer kompensatorische Sinn-stiftungen (exakt so lautet die Ökonomie in Hofmannsthals Brief; für sein dumpfes Vor-sich-hin-Existieren wird Lord Chandos mit Lichtblicken entschädigt). Eine solche Integration der Epiphanie in das System, scheint es, wäre nun geradezu ein Frevel am "Stern der Sinnlosigkeit" ${ }^{\text {"19. }}$. Das Bemerkenswerte an den Obèriuten gegenüber dem ,hohen 'Modernismus eines Hugo von Hofmannsthal - oder auch gegenüber den poetischen Sophiologien des russischen Silbernen Zeitalters - liegt darin, dass sie keine säkularisierten Erleuchtungen benötigen. Vvedenskij und Charms kommen ohne Kompensationslogik aus. Andererseits kennen sie auch keine ,Lust' an der Dekomposition der alten Welt wie die frühen, analytischen Avantgarden. Was ihnen vorschwebt, ist eine reale Bezeugung der Dekomposition der Welt und damit ein Aufweis ihrer Erlösungsbedürftigkeit. (In Daniil Charms Tagebuch werden scheinbar nihilistische und nicht selten libertine Notizen immer wieder von Gebeten und Sprüngen in den Glauben unterbrochen.)

Das fragmentarische Graue Heft bietet eine schillernde Philosophie der Zeit, und ihr Einsatz erinnert stark an Henri Bergson. Vvedenskij geht es darum, ein reduktiv räumlichgegenständliches Wirklichkeitsverständnis zu widerlegen. Jedes Absehen von der Zeitlichkeit bedeute ein Verfehlen der Wirklichkeit überhaupt, da gerade die Zeit die Wirklichkeit ausmache. Hier setzt auch Vvedenskijs Sprachkritik an: Die Sprache könne nur Starr-Raumgreifendes ausdrücken („die Härte des Steins / überzeugt mich von nichts mehr“, „alles alles hat Länge, / hat Breite“; Vvedenskij 1993d: 76), daher „entspricht“ (sootvetstvuet) sie der Zeit nicht (Vvedenskij 1993d: 79). Die Sprache ist in diesem Sinne unrealistisch, oder vielleicht adäquater ausgedrückt: irreal. Nur die Zeit gibt es. Genau deshalb ist sie nicht sprachlich objektivierbar. Das Werk der Zeit ist jenes der Annullierung des Seienden:

Die Zeit ist das einzige, was außerhalb von uns nicht existiert. Sie verschluckt alles Seiende außerhalb von uns. Eine Nacht der Vernunft stellt sich ein. Die Zeit geht über uns auf wie ein Stern. Werfen wir unsere Denkköpfe ab, d. h. unsere Vernünfte. Schaut sie wurde sichtbar. Sie geht über uns auf wie die Null. Sie verwandelt alles in Null. (Vvedenskij 1993d: 78-79)

Ist die Zeit Gott? Oder ist sie eher das, was die Ankunft Gottes ,aufhält'? Wenn es weiter heißt (Vvedenskij 1993d: 82): „Wilde Tiere die Zeit sitzt über euch. Die Zeit denkt an euch, und Gott [denkt an euch]", scheinen Zeit und Gott geradezu zusammenzufallen. Doch anschließend an die Passage zur alles verschluckenden Zeit heißt es (Vvedenskij 1993d: 79): „(Die letzte Hoffnung - Christus ist auferstanden.) / Christus ist auferstanden - die letzte Hoffnung.“ Die Auferstehung markiert nun eine Gegenperspektive zur ,aufgehenden Zeit“. Dabei wäre die Auferstehung gerade kein die Zeit unterbrechendes epiphanisches Geschehen. Die Zeit ist zu mächtig, als dass sie von einer subjektiven Gewissheit suspendiert

19 Vgl. folgende Stelle aus Ringsum ist womöglich Gott (Krugom vozmožno Bog, 1931; Vvedenskij 1993a: 152), auf die sich die oben (Fußnote 14) erwähnte Arbeit Jakov Druskins bezieht: „Es leuchtet der Sinnlosigkeit Stern [bessmyslicy zvezda], / sie allein ist ohne Boden [bezdna]." 
werden könnte, und sei es für einen Augenblick. „Christus ist auferstanden“ indiziert vielmehr die Hoffnung auf eine fundamental andere Zeit. ${ }^{20}$ So aber lässt sie das Empfinden der sich ausbreitenden „Null“ hier und jetzt nur noch nackter zur Geltung kommen.

Ganz bergsonistisch bemerkt Vvedenskij (1993d: 81): „[...] Du hast Bewegung und Zeit mit Raum verwechselt. Du hast sie irrtümlicherweise übereinander gelegt [...].“ Solange man im Lauf einer Maus lediglich ihre Fortbewegung durch den Raum sehe, verfehle man das Wesen der Zeit. Erst wenn sich der zielgerichtete Laufschritt der Maus vor unseren Augen zu fragmentieren beginne, werde - im rasenden Stillstand der Null - die Zeit sichtbar als „Flimmern“ (mercanie): „Die Maus beginnt zu flimmern. Siehe: die Welt flimmert (wie eine Maus).“(Vvedenskij 1993d: 81) Die Zeit wäre also ein schwingender „Punkt“ (točka), der „stille Rumpf“ (tichoe tulovišce) der Dinge, das eigentlich Reale an ihnen. Anders als bei Bergson (s. Kołakowski 2008: 26-28) ist die Zeit bei Vvedenskij gerade kein quasi melodiöses Kontinuum. Denn: Ihr Sitz ist nicht das Gedächtnis. Die Zeit ist hier, in deutlicher Abweichung von dem Mnemoniker Bergson, gedächtnislos: „Ich vertraue nicht dem Gedächtnis, ich glaube nicht der Vorstellung" (Vvedenskij 1993d: 78). Dieser unbergsonistische Bergsonismus ist für das Schreiben einer ,katastrophalen Moderne' in höchstem Maße bezeichnend.

Vvedenskij gibt im Grauen Heft an, die Zeit erstmals "gefühlt" zu haben, als er im Gefängnis war (er wurde 1931 wegen „konterrevolutionärer Gesinnung“ festgenommen und 1932 von Leningrad nach Kursk verbannt; 1941 kam er nach neuerlicher Festnahme bei einem Zugtransport ums Leben). Die im Gefängnis ohne äußere Ereignisse ,rein` erlebte Zeit befreie von den Umrissen der objektivierten Wirklichkeit. Doch zugleich bestraft sie ihren Neophyten („Die Bestrafung erhielt ich auch in Form von Zeit“; Vvedenskij 1993d: 83) mit „wildem Nicht-Verstehen“ (dikoe neponimanie). „[...] diesem Nicht-Verstehen“, lesen wir (Vvedenskij 1993d: 79), „wird niemand jemals etwas Klares gegenüberstellen können““

Die Epiphanie müsste letztlich ein Moment der Klarsicht sein. So bestätigt sich noch einmal, dass es für Vvedenskij eben keine solche Klarsicht gibt, durch die das Nicht-Verstehen im Sinne epiphanischer Plötzlichkeit aufgewogen werden könnte. Die Auferstehung, d. h. die Hoffnung auf eine radikal andere Zeit, eine wahrhaft „neue Sekunde“ (Vvedenskij 1993d: 83), steht auf einem anderen Blatt als alles, was literarische Epiphanien zu leisten vermögen.

Wenn wir nun einen Schritt von diesen zeitphilosophischen Ausführungen machen und an Vvedenskijs Gedichte und Kurzdramen denken, so stellt sich die Frage: Ist seine poetische Praxis denn nicht eigentlich durch und durch - epiphanisch? Ist sein Schreiben nicht geradezu ein Schreiben des reinen Scheins? Was wenn nicht ein reines Erscheinen zeigen Passagen wie diese aus Ringsum ist womöglich Gott (Krugom vozmožno Bog, 1931):

Herr, wie ist die Welt wundervoll,

wie ist in der Welt alles gut,

Ich halte Andacht für die Götter,

ich werde zerrieben zu Pulver

20 Vgl. Ilja Kukujs (2008: 83) Ausführungen zum „Tod Gottes“ bei Vvedenskij, der am ehesten „diesseitig“ zu verstehen sei. Als schlechthin „seiender“, dadurch aber ganz und gar jenseitiger ist Gott in Vvedenskijs poetischer Welt keineswegs „tot“; vgl. die Aussage am Ende von Ringsum ist womöglich Gott (Vvedenskij 1993a: 152): „Sein kann nur Gott“" (Byt' možet tol'ko Bog). 
angesichts so machtvoller, so geheimniserfüllter Dinge, die vorbeiziehen auf den Wolken im Bild einer Packung Kerzen. Mein Gott, alles in der Welt ist üppig, Prachtvoll und geistreich.

$\mathrm{Zu}$ Gott beten unhörbar Meer, Elch, Krug, Tenne,

Kerze, Reiter, Mensch,

Löffel und Chadschi-Abrek.

(Vvedenskij 1993a: 130)

Tatsächlich, es geht hier nicht um die Substazialität des Erscheinenden. Man kann im (Sprach-) Geschehen dieser Verse durchaus einen „Augenblick des ästhetischen Scheins“(Bohrer) sehen, der den Sprechenden zu einer Rhetorik des Götterlobs regelrecht zwingt. Ob die Götter dabei eine Metapher darstellen wie etwa - nach Bohrer (1998 [1981]: 263) - in Hölderlins Oden, ist nicht entscheidend, denn selbst falls sie es sind, könnte kaum näher gesagt werden, wofür die Metapher steht. Entscheidend ist vielmehr die evidente Wahllosigkeit oder genauer: die Inkompetenz des Lobes. Wer ist der Sprecher? Es ist ein Sperling, „die Körnchen der Freude pickend“, während sich Menschen zum Spektakel einer Hinrichtung zusammenfinden (Vvedenskij 1993a: 130). Das Tier (zver') hat bei Vvedenskij eine privilegierte Stellung. „Es tut mir leid, dass ich kein Tier bin“"21, „Mit Neid schaue ich auf das Tier “22, „Tiere ihr seid Glocken“(Vvedenskij 1993d: 82), lauten nur einige seiner einschlägig ,animalischen' Verse. Vom Sperlingsgebet in Ringsum ist womöglich Gott her argumentierend, ließe sich sagen: Das wilde Tier - und gerade nicht der irrende Mensch - ist in Vvedenskijs poetischer Welt als zur Epiphanie hin offen bestimmt. ${ }^{23} \mathrm{Im}$ Fall des betenden Spatzen ist dabei ein stark sarkastischer Beiklang nicht zu überhören. Und doch spricht der Vogel, zwar exaltiert oder gar ins Lächerliche gewendet, Vvedenskijs poetisches Idiom, so dass man in seinem Gebet noch immer einen Ausdruck des übergeordneten „alles wird unverständlich“(Vvedenskij 1993d: 83) sehen muss - kaum jedoch einen Moment subjektiver Aufhellung.

Wenn in der ,klassischen' epiphanischen Moderne sich mitten in der Sprachkrise eine neue Weltaneignung und ein neuer Vorstoß zu verborgenen Strukturen abzeichnen (wie in Hofmannsthals Brief), so zieht sich die Welt in der nicht-mehr-epiphanischen Poetik Vvedenskijs vollständig ins Verborgene zurück. Aber ihre Verborgenheit liegt offen zu Tage.

21 Vvedenskij 1993b [1934]. Für eine deutsche Übersetzung s. Urban 1992a: 20-22.

2 Vvedenskij 1993c [1940]: 68. Für eine deutsche Übersetzung s. Vvedenskij 2013a.

23 An der Figur des ,Tieres' könnte noch einmal Vvedenskijs typologische Nähe und Distanz zu Hofmannsthal aufgewiesen werden: Im Gespräch über Gedichte (1904) bemerkt einer der Dialogpartner, Tiere seien „die eigentlichen Hieroglyphen, [...] lebendige geheimnisvolle Chiffren, mit denen Gott unaussprechliche Dinge in die Welt geschrieben hat" (Hofmannsthal 1991b: 79). 


\section{Literatur}

Abril, Henri (Hg.) (2012): La baignoire d'Archimède. Anthologie poétique de l'Obèriou. Belval: Circé. Barskova, Polina (2011): Vvedenskij i „my“ [Vvedenskij und „wir"]. In: Novoe literaturnoe obozrenie (2), 222-224.

Bohrer, Karl Heinz (1998 [1981]): Plötzlichkeit. Zum Augenblick des ästhetischen Scheins. Mit einem neuen Nachwort von 1998. Frankfurt a. M.: Suhrkamp.

de Man, Paul (1960): Structure intentionnelle de l'Image romantique. In: Revue internationale de Philosophie (14/51,1), 68-84.

Druskin, Jakov (2000 [1973]): Zvezda bessmyslicy. In: Valerij Sažin (Hg.): Sborišče druzej, ostavlennych sud'boju. „Činari“ v tekstach, dokumentach i issledovanijach v dvuch tomach [Eine Ansammlung Freunde, die vom Schicksal verlassen wurden. Die „Činari“ in Texten, Dokumenten und Untersuchungen in zwei Bänden]. Bd. 1. Moskva: Ladomir, 323-416.

Gerasimova, Anna (2011): Ob Aleksandre Vvedenskom [Über Aleksandr Vvedenskij]. In: Aleksandr Vvedenskij, $V_{s e}[$ Alles]. Hg. Anna Gerasimova. Moskva: OGI, 7-24.

Günther, Timo (2004): Hofmannsthal: Ein Brief. München: Fink.

Hansen-Löve, Aage A. (1989): Der Russische Symbolismus. System und Entfaltung derpoetischen Motive. Bd. 1: Diabolischer Symbolismus. Wien: Verlag der Österreichischen Akademie der Wissenschaft.

Hansen-Löve, Aage A. (1994): Konzepte des Nichts im Kunstdenken der russischen Dichter des Absurden (Obèriu). In: Poetica 26 (3/4), 308-373.

Hansen-Löve, Aage A. (1998): „Scribo quia absurdum“. Die Religionen der russischen Dichter des Absurden (Obèriu). In: Maria Deppermann (Hg.): Russisches Denken im europäischen Dialog. Innsbruck: Studien Verlag, 160-203.

Hofmannsthal, Hugo von (1991): Sämtliche Werke. Bd. XXXI. Erfundene Gespräche und Briefe. Hg. Ellen Ritter. Frankfurt a. M.: Fischer.

Hofmannsthal, Hugo von (1991a): Ein Brief. In: Hofmannsthal 1991: 45-55.

Hofmannsthal, Hugo von (1991b): Das Gespräch über Gedichte. In: Hofmannsthal 1991: 74-86.

Ičin, Kornelija / Kudrjavcev, Sergej (Hgg.) (2006): Poèt Aleksandr Vvedenskij. Sbornik materialov [Der Dichter Aleksandr Vvedenskij. Sammelband]. Belgrad: Gileja.

Jean-Philippe Jaccard (1991): Danill Harms et la fin de l'avant-garde russe. Bern etc.: Peter Lang.

Kling, Oleg (1999): Évoljucija i „latentnoe“ suščestvovanie simvolizma posle Oktjabrja [Die Evolution und „latente“ Existenz des Symbolismus nach der Oktoberrevolution]. In: Voprosy literatury 4, 37-64.

Kovalenko, Georgij (Hg.) (2003): Simvolizm v avangarde [Symbolismus in der Avantgarde]. Moskva: Nauka.

Kołakowski, Leszek (2008): Bergson. Kraków: Universitas.

Kukuj, Il'ja (2008): Kto molča udaljaet vremja? (Zametki ob odnom personaže Aleksandra Vvedenskogo) [Wer entfernt schweigend die Zeit? Anmerkungen zu einer Figur bei Aleksandr Vvedenskij]. In: Russica Romana 15, 73-84.

Kühn, Joachim (1975): Gescheiterte Sprachkritik. Fritz Mauthners Leben und Werk. Berlin/New York: De Gruyter.

Lehmann, Gudrun (2013): Franz Kafka und Daniil Charms. Versuch einer Annäherung. In: Zeitschrift für Slawistik 58(3), 276-296. 
Lipavskij, Leonid (2000 [1933/34]): Razgovory. In: Valerij Sažin (Hg.): Sborišče druzej, ostavlennych sud'boju. „Činari“"v tekstach, dokumentach i issledovanijach $v$ dvuch tomach [Eine Ansammlung Freunde, die vom Schicksal verlassen wurden. Die „Činari“ in Texten, Dokumenten und Untersuchungen in zwei Bänden]. Bd. 1. Moskva: Ladomir, 174-254.

Majakovskij, Vladimir (1959 [1922]): V. V. Chlebnikov. In: Ders.: Polnoe sobranie sočinenij v trinadcati tomach [Gesamtausgabe in 13 Bänden]. Bd. 12. Moskva: Chudožestvennaja literatura, 23-28.

Majewski, Paweł (2018): Dwie próby o literaturze: Stowacki i Hofmannsthal [Zwei Versuche über Literatur: Słowacki und Hofmannsthal]. Warszawa: Convivo.

Martynova, Ol'ga (2019 [2008]): Oda na užas [Ode auf den Schrecken]. In: Oleg Jur'ev (Hg.): Leningradskaja chrestomatija (ot pereimenovanija do pereimenovanija). Malen'kaja antologija velikich leningradskich stichov. Sankt-Peterburg: Izdatel'stvo Ivana Limbacha (im Erscheinen).

Merger, Andrea (1995): Becketts Rhetorik des Sprachmißbrauchs. Heidelberg: Winter.

Müller, Bertram (1978): Absurde Literatur in Russland. München: Sagner.

Nycz, Ryszard (2001): Literatura jako trop rzeczywistości. Poetyka epifanii w nowoczesnej literaturze polskiej [Literatur als Trope der Wirklichkeit. Epiphanische Poetik in der modernen polnischen Literatur]. Kraków: Universitas.

Ostashevsky, Eugene (Hg.) (2006): Oberiu: An Anthology of Russian Absurdism. Evanston, IL: Northwestern University Press.

Podoroga, Valerij (1993): Beseda s V. Podorogoj. K voprosu o mercanii mira [Gespräch mit V. Podoroga. Zur Frage des Flimmerns der Welt]. In: Logos 4, 139-150.

Saße, Günter (1977): Sprache und Kritik. Untersuchungen zur Sprachkritik der Moderne. Göttingen: Vandenhoeck und Ruprecht.

Sauerwald, Lisanne (2010): Mystisch-hermetische Aspekte im Kunstdenken der russischen Dichter des Absurden. Würzburg: Ergon.

Sažin, Valerij (Hg.) (2000): Sborišče druzej, ostavlennych sud'boju. „Činari“v tekstach, dokumentach $\mathrm{i}$ issledovanijach v dvuch tomach [Eine Ansammlung Freunde, die vom Schicksal verlassen wurden. Die „Činari“ in Texten, Dokumenten und Untersuchungen in zwei Bänden]. Bd. I. Moskva: Ladomir.

Schloßberger-Oberhammer, Anja (2018): Leonid Lipavskijs Gedankenwelten. Zumparadoxalen Denken der russischen Dichter des Absurden [Wiener Slawistischer Almanach - Sonderband 95]. Berlin: Peter Lang.

Schultz, H. Stefan (1961): Hofmannsthal and Bacon: The Sources of the Chandos Letter. In: Comparative Literature (13/1), 1-15.

Skidan, Aleksandr (2013): Pre-vraščenie: Poètičeskie mašiny Aleksandra Vvedenskogo [Um-kehrung: Aleksandr Vvedenskijs poetische Maschinen]. In: Žan-Filipp Žakkar, Violen Fridli, Jens Cherl't (Hgg.): „Vtoraja kul'tura“. Neoficial'naja poèzija Leningrada v 1970-1980-e gody. Sankt-Peterburg: Rostok, 53-73.

Tokarev, Dmitrij (2002): Kurs na chudšee. Absurd kak kategorija teksta u Daniila Charmsa i Semjuelja Bekketa [Kurs aufs Schlimmste. Das Absurde als Textkategorie bei Daniil Charms und Samuel Beckett]. Moskva: Novoe literaturnoe obozrenie.

Urban, Peter (Hg.) (1992a): „Oberiu - Vereinigung der realen Kunst. Topographie einer literarischen Landschaft [Teil I]“. In: Schreibheft 39. 
Urban, Peter (Hg.) (1992b): „Oberiu - Vereinigung der realen Kunst. Topographie einer literarischen Landschaft [Teil II]“. In: Schreibheft 40.

Valieva, Julija (2007): Igra v bessmyslicu. Poètičeskij mir Aleksandra Vvedenskogo [Spiel mit der Sinnlosigkeit. Die poetische Welt Aleksandr Vvedenskijs]. Sankt-Peterburg: Dmitrij Bulanin.

Vvedenskij, Aleksandr (1993): Polnoe sobranie proizvedenij v dvuch tomach [Gesamtausgabe in zwei Bänden]. Hg. M. Mejlach. Moskva: Gileja.

Vvedenskij, Aleksandr (1993a): Krugom vozmožno Bog [Ringsum ist womöglich Gott]. In: Polnoe sobranie proizvedenij $v$ dvuch tomach [Gesamtausgabe in zwei Bänden]. Hg. Michail Mejlach. Bd. 1. Moskva: Gileja, 127-152.

Vvedenskij, Aleksandr (1993b): Mne žalko chto ja ne zver’... [Es tut mir leid, dass ich kein Tier bin...] In: Polnoe sobranie proizvedenij v dvuch tomach [Gesamtausgabe in zwei Bänden]. Hg. Michail Mejlach. Bd. 2. Moskva: Gileja, 183-185.

Vvedenskij, Aleksandr (1993c): Ėlegija [Elegie]. In: Polnoe sobranie proizvedenij v dvuch tomach [Gesamtausgabe in zwei Bänden]. Hg. Michail Mejlach. Bd. 2. Moskva: Gileja, 68-69.

Vvedenskij, Aleksandr (1993d): Seraja tetrad' [Das graue Heft]. In: Polnoe sobranie proizvedenij v dvuch tomach [Gesamtausgabe in zwei Bänden]. Hg. Michail Mejlach. Bd. 2. Moskva: Gileja, 75-85.

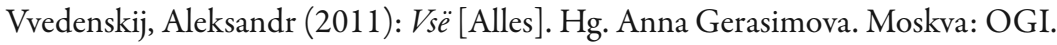

Vvedenskij, Aleksandr (2013a): Elegie. In: Akzente (60/4), 344-346.

Vvedenskij, Aleksandr (2013b): An Invitation for Me to Think. Hg. und übers. Eugene Ostashevsky. New York: New York Review Books.

Zabolockij, Nikolaj (2011 [1926]): Moi vozraženija A. I. Vvedenskomu, avtoritetu bessmyslicy [Meine Einwände gegen A.I Vvedenskij, Autorität der Sinnlosigkeit]. In: Aleksandr Vvedenskiij: Vsë [Alles]. Hg. Anna Gerasimova. Moskva: OGI, 393-395.

Zehnder, Christian (2013): Aleksandr Vvedenskij: Schreckliche Schönheit. In: Akzente (60/4), 347-349.

Zimmermann, Tanja (2007): Abstraktion und Realismus im Literatur-und Kunstdiskurs der russischen Avantgarde [Wiener Slawistischer Almanach - Sonderband 68]. Wien/München: Sagner. 